\title{
On the Patterns of Principal Curvature Lines around a Curve of Umbilic Points
}

\author{
RONALDO GARCIA ${ }^{1}$ and JORGE SOTOMAYOR ${ }^{2}$ \\ ${ }^{1}$ Instituto de Matemática e Estatística, Universidade Federal de Goiás \\ Caixa Postal 131 - 74001-970 Goiânia, GO, Brasil \\ ${ }^{2}$ Instituto de Matemática e Estatística, Universidade de São Paulo \\ Rua do Matão 1010, Cidade Universitária, 05508-090 São Paulo, SP, Brasil
}

Manuscript received on June 15, 2004; accepted for publication on October 10, 2004; contributed by JORGE SOTOMAYOR*

\begin{abstract}
In this paper is studied the behavior of principal curvature lines near a curve of umbilic points of a smooth surface.
\end{abstract}

Key words: Umbilic point, principal curvature lines, principal cycles.

\section{INTRODUCTION}

The study of umbilic points on surfaces and the patterns of principal curvature lines around them has attracted the attention of generation of mathematicians among whom can be named Monge, Darboux and Carathéodory. One aspect - concerning isolated umbilics - of the contributions of these authors, departing from Darboux (Darboux 1896), has been elaborated and extended in several directions by Garcia, Sotomayor and Gutierrez, among others. See (Gutierrez and Sotomayor, 1982, 1991, 1998), (Garcia and Sotomayor, 1997, 2000) and (Garcia et al. 2000, 2004) where additional references can be found.

In (Carathéodory 1935) Carathéodory mentioned the interest of non isolated umbilics in generic surfaces pertinent to Geometric Optics. In a remarkably concise study he established that any local analytic regular arc of curve in $\mathbb{R}^{3}$ is a curve of umbilic points of a piece of analytic surface. In some cases he also determined the patterns of behavior of principal curvature lines near the curve of umbilic points.

In the present paper will be performed an analytic, explicit and constructive study of umbilic curves and of the simplest patterns for their neighboring principal curvature lines, that holds also

*Member Academia Brasileira de Ciências

Correspondence to: ragarcia@mat.ufg.br / sotp@ime.usp.br 
for smooth curves and surfaces. A comparison of results of this work with those of Carathéodory (Carathéodory 1935) is attempted in section 6.

\section{PRELIMINARIES}

Let $c:[0, l] \rightarrow \mathbb{R}^{3}$ be a regular curve parametrized by arc length $u$ contained in a regular smooth surface $\mathbb{M}$, which is oriented by the once for all given positive unit normal vector field $N$.

Let $T \circ c=c^{\prime}$. According to (Spivak 1980), the Darboux frame $\{T, N \wedge T, N\}$ along $c$ satisfies the following system of differential equations:

$$
\begin{aligned}
T^{\prime} & =k_{g} N \wedge T+k_{n} N \\
(N \wedge T)^{\prime} & =-k_{g} T+\tau_{g} N \\
N^{\prime} & =-k_{n} T-\tau_{g}(N \wedge T)
\end{aligned}
$$

where $k_{n}$ is the normal curvature, $k_{g}$ is the geodesic curvature and $\tau_{g}$ is the geodesic torsion of the curve $c$.

Proposition 1. Let $c:[0, l] \rightarrow \mathbb{M}$ be a regular arc length parametrization of a curve of umbilic points, such that $\{T, N \wedge T, N\}$ is a positive frame of $\mathbb{R}^{3}$. Then the expression

$$
\alpha(u, v)=c(u)+v(N \wedge T)(u)+\left[\frac{1}{2} k(u) v^{2}+\frac{1}{6} a(u) v^{3}+\frac{1}{24} b(u) v^{4}+\text { h.o.t }\right] N(u),
$$

where $k(u)=k_{n}(c(u), T)=k_{n}(c(u), N \wedge T)$ is the normal curvature of $\mathbb{M}$ in the directions $T$ and $N \wedge T$, defines a local $C^{\infty}$ chart in a small tubular neighborhood of $c$. Moreover $\tau_{g}(u)=0$.

PROOF. This parametrization, in the case where $c$ is a principal curvature line, was first introduced by Gutierrez and Sotomayor in (Gutierrez and Sotomayor 1982). In the present case, $c$ being a regular curve of umbilic points, by the Implicit Function Theorem it follows that the principal curvatures $k_{1} \leq k_{2}$ restricted to $c$ are also $C^{\infty}$. Also, it follows that $k_{n}(c(u), T(u))=k_{n}(c(u),(N \wedge T)(u))$ and in consequence $\tau_{g}(c(u), v)=0$ for any $v \in T_{c(u)} \mathbb{M}$.

\section{FIRST AND SECOND FUNDAMENTAL FORMS}

In the chart $\alpha$ in the equation (2) the positive unit normal vector vector field is given by

$$
N=\frac{\alpha_{u} \wedge \alpha_{v}}{\left|\alpha_{u} \wedge \alpha_{v}\right|}
$$

So it follows that,

$$
\begin{aligned}
N(u, v)=- & {\left[\frac{1}{2} k^{\prime} v^{2}+\frac{1}{6}\left(a^{\prime}+3 k^{\prime} k_{g}\right) v^{3}+0\left(v^{4}\right)\right] T(u) } \\
& -\left[k v+\frac{1}{2} a(u) v^{2}+\frac{1}{6}\left(b(u)-3 k^{3}\right) v^{3}+0\left(v^{4}\right)\right](N \wedge T)(u) \\
& +\left[1-\frac{1}{2} k^{2} v^{2}+\frac{1}{2} k^{\prime} k v^{3}+O\left(v^{4}\right)\right] N(u)
\end{aligned}
$$


The coefficients of the first and second fundamental forms in the chart $\alpha$ are given by: $E=<\alpha_{u}, \alpha_{u}>, \quad F=<\alpha_{u}, \alpha_{v}>, \quad G=<\alpha_{v}, \alpha_{v}>, \quad e=<\alpha_{u u}, N>, \quad f=<\alpha_{u v}, N>$ and $g=<\alpha_{v v}, N>$. Therefore,

$$
\begin{aligned}
E(u, v) & =1-2 k_{g} v+\left(k_{g}^{2}-k^{2}\right) v^{2}+\frac{1}{6}\left(6 k_{g} k^{2}-2 k a(u)\right) v^{3}+O\left(v^{4}\right) \\
F(u, v) & =\frac{1}{2} k^{\prime} k v^{3}+O\left(v^{4}\right) \\
G(u, v) & =1+k^{2} v^{2}+k a(u) v^{3}+O\left(v^{4}\right) \\
e(u, v) & =k-2 k_{g} k v+\frac{1}{2}\left(2 k k_{g}^{2}-k_{g} a(u)-2 k^{3}+k^{\prime \prime}\right) v^{2} \\
& +\frac{1}{6}\left[a^{\prime \prime}+k_{g}\left(9 k^{3}-b(u)\right)+\left(3 k_{g}^{2}-k^{2}\right) a(u)\right. \\
& \left.+3 k^{\prime}\left(k_{g}^{\prime}+k^{2}\right)\right] v^{3}+O\left(v^{4}\right) \\
f(u, v) & =k^{\prime} v+\frac{1}{2}\left(k_{g} k^{\prime}+a^{\prime}\right) v^{2}+\frac{1}{6}\left(k_{g} a^{\prime}+3 k^{\prime} k_{g}^{2}+b^{\prime}\right) v^{3}+O\left(v^{4}\right) \\
g(u, v) & =k+a(u) v+\frac{1}{2}\left(b(u)-k^{3}\right) v^{2}-\frac{1}{2} k^{2}\left(a(u)-k^{\prime}\right) v^{3}+O\left(v^{4}\right)
\end{aligned}
$$

The Mean and Gauss curvatures in the chart $\alpha$ are given by:

$$
\begin{aligned}
\mathcal{H} & =k+\frac{1}{2} a(u) v+\frac{1}{4}\left(b(u)+k^{\prime \prime}-3 k^{3}-k_{g} a(u)\right) v^{2}+O\left(v^{3}\right) \\
\mathcal{K} & =k^{2}+k a(u) v+\frac{1}{2}\left(-k_{g} k a(u)-3 k^{4}+k k^{\prime \prime}+k b(u)-2 k^{\prime \prime}\right) v^{2}+O\left(v^{3}\right)
\end{aligned}
$$

According to (Spivak 1980) the differential equation of curvature lines in the chart $\alpha$ is given by

$$
\begin{aligned}
& (F g-G f) d v^{2}+(E g-G e) d u d v+(E f-F e) d u^{2}= \\
& =L d v^{2}+M d v d u+N d u^{2}=0 ; \quad \text { where } \\
L & =-\left[k^{\prime} v+\frac{1}{2}\left(k_{g} k^{\prime}+a^{\prime}\right) v^{2}+\frac{1}{6}\left(k_{g} a^{\prime}+3 k^{\prime} k_{g}^{2}+b^{\prime}+3 k^{2} k^{\prime}\right) v^{3}+O\left(v^{4}\right)\right] \\
M & =a(u) v+\frac{1}{2}\left[b(u)-3 k^{3}-k^{\prime \prime}-3 k_{g} a(u)\right] v^{2} \\
& +\frac{1}{6}\left[15 k^{3} k_{g}-3 k_{g}^{\prime} k^{\prime}+\left(3 k_{g}^{2}-16 k^{2}\right) a(u)-a^{\prime \prime}-5 k_{g} b(u)\right] v^{3}+O\left(v^{4}\right) \\
N & =k^{\prime} v+\frac{1}{2}\left(a^{\prime}-3 k_{g} k^{\prime}\right) v^{2}+\frac{1}{6}\left(3 k^{\prime} k_{g}^{2}-9 k^{2} k^{\prime}-5 k_{g} a^{\prime}+b^{\prime}\right) v^{3}+O\left(v^{4}\right)
\end{aligned}
$$

\section{PRINCIPAL CONFIGURATIONS NEAR AN UMBILIC CURVE}

Proposition 2. Suppose that $\nabla \mathcal{H}(u, 0)=\left(k^{\prime}, a(u) / 2\right)$ is not zero at a point $u_{0}$. Then the principal foliations near the point $\left.c\left(u_{0}\right)\right)$ of the curve are as follows.

i) If $k^{\prime}\left(u_{0}\right) \neq 0$ then both principal foliations are transversal to the curve of umbilic points. See Fig. 1, left. 
ii) If $k^{\prime}\left(u_{0}\right)=0, k^{\prime \prime}\left(u_{0}\right) \neq 0$ and $a\left(u_{0}\right) \neq 0$, then one principal foliation is transversal to $c$ and the other foliation has quadratic contact with the curve $c$ at the point $c\left(u_{0}\right)$. See Fig. 1, center and right.
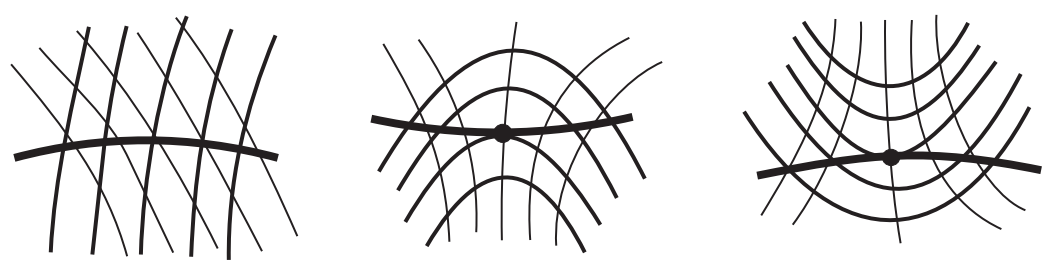

Fig. 1 - Principal curvature lines near an umbilic curve: transversal case, left, and tangential case, center and right.

Proof. After division by $v$, the implicit differential equation of curvature lines is given by:

$$
\begin{aligned}
\mathcal{F}(u, v,[d u: d v])= & -\left[k^{\prime}+\frac{1}{2}\left(k_{g} k^{\prime}+a^{\prime}\right) v+O\left(v^{2}\right)\right] d v^{2} \\
& +\left[a(u)+\frac{1}{2}\left(b(u)-3 k^{3}-k^{\prime \prime}-3 k_{g} a(u)\right) v+O\left(v^{2}\right)\right] d u d v \\
& +\left[k^{\prime}+\frac{1}{2}\left(a^{\prime}-3 k_{g} k^{\prime}\right) v+O\left(v^{2}\right)\right] d u^{2}=0,
\end{aligned}
$$

Consider the Lie-Cartan line field $X=\left(\mathcal{F}_{p}, p \mathcal{F}_{p},-\left(\mathcal{F}_{u}+p \mathcal{F}_{v}\right)\right)$ where $\mathcal{F}=L p^{2}+M p+N=$ $0, p=d v / d u$. It follows that $\mathcal{F}(u, 0, p)=k^{\prime}\left(1-p^{2}\right)+a(u) p$ and so when $k^{\prime} \neq 0, \mathcal{F}(u, v, p)=0$ is a regular surface near $v=0$ and has two connected components containing the points $\left(u, 0, p_{ \pm}\right)$, where $p_{ \pm}=\left[a(u) \pm \sqrt{a^{2}+4\left(k^{\prime}\right)^{2}}\right] /\left(2 k^{\prime}\right) \neq 0$. Clearly the Lie-Cartan line field field $X$ is regular at $\left(0,0, p_{ \pm}\right)$and so the configuration of principal lines is as shown in Fig. 1, left.

Suppose for simplicity that at $u=0$ it holds that $k^{\prime}(0)=0$. By hypothesis, we have that $a(0)=a_{0} \neq 0$. Then, after division by $v$, it follows that the implicit differential equation of principal curvature lines is given by:

$$
\begin{aligned}
\mathcal{F}= & -\left[k^{\prime \prime}(0) u+\frac{1}{2} a^{\prime}(0) v+\cdots\right] d v^{2} \\
& +\left[a_{0}+a^{\prime}(0) u+\left(b(0)-3 k(0)^{3}-k^{\prime \prime}-3 k_{g}(0) a_{0}\right) v+\cdots\right] d v d u \\
& +\left[k^{\prime \prime}(0) u+\frac{1}{2} a^{\prime}(0) v+\cdots\right] d u^{2}=0
\end{aligned}
$$

Therefore the directions defined by $\mathcal{F}(u, v,[d u: d v])=0$ are $p=0$ and $q=0$ where $p=d v / d u$ and $q=d u / d v$. Therefore one direction is tangent to $c$ and the other is orthogonal to $c$.

By the conditions imposed, one foliation is orthogonal to, and the other has quadratic contact with, the umbilic curve at $c(0)$. 
In fact, $\mathcal{F}_{p}(0,0,0)=a_{0} \neq 0$ and it follows that the integral curve of the Lie-Cartan line field $X=\left(\mathcal{F}_{p}, p \mathcal{F}_{p},-\left(\mathcal{F}_{u}+p \mathcal{F}_{v}\right)\right)$ passing through $(0,0,0)$ is given by:

$$
\begin{aligned}
& u(t)=a_{0} t+\cdots \\
& v(t)=-\frac{1}{2} k^{\prime \prime}(0) a_{0} t^{2}+\cdots \\
& p(t)=-k^{\prime \prime}(0) t+\ldots
\end{aligned}
$$

The curve $(u(t), v(t))$ has quadratic contact with the line $v=0$, provided $k^{\prime \prime}(0) a_{0} \neq 0$. Eliminating $t$ it follows that $v=-\frac{k^{\prime \prime}}{2 a_{0}} u^{2}+\cdots$.

To describe the other principal foliation near $(0,0,0)$ it is convenient to consider the Lie-Cartan line field $Y=\left(q \mathcal{F}_{q}, \mathcal{F}_{q},-\left(q \mathcal{F}_{u}+\mathcal{F}_{v}\right)\right)$ with $q=d u / d v$. It is clear that this principal foliation is transversal to $c$ in a neighborhood of $c(0)$. Therefore the principal configuration is as shown in Fig. 1, center and right. This ends the proof.

Proposition 3. Suppose that $k^{\prime}(0)=a(0)=0, a^{\prime}(0) k^{\prime \prime}(0) \neq 0$, at the point $c(0)$ of a regular curve $c$ of umbilic points. Let $A:=-2 k^{\prime \prime}(0) / a^{\prime}(0) \neq 0$ and $B:=\left[b(0)-3 k(0)^{3}-k^{\prime \prime}(0)\right] / a^{\prime}(0)$. Let $\Delta$ and $\delta$ be defined by

$$
\Delta=-4 A^{4}+12 B A^{3}-\left(36+12 B^{2}\right) A^{2}+\left(4 B^{3}+72 B\right) A-9 B^{2}-108 ; \quad \delta=2-A B .
$$

Then the principal foliations at this point are as follows.

i) If $\delta<0$ and $\Delta<0$ then 0 is topologically equivalent to a Darbouxian umbilic of type $D_{1}$, through which the umbilic curve is adjoined transversally to the separatrices. See Fig. 2 left.

ii) If $\delta<0$ and $\Delta>0$ then 0 is topologically equivalent to a Darbouxian umbilic of type $D_{2}$, through which the umbilic curve is adjoined, on the interior of the parabolic sectors, transversally to the separatrices and to the nodal central line. See Fig. 2 center.

iii) If $\delta>0$ then 0 is topologically a Darbouxian umbilic of type $D_{3}$, through which the umbilic curve is adjoined transversally to the separatrices. See Fig. 2 right.

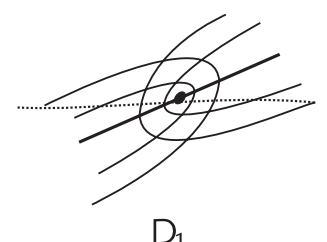

$\mathrm{D}_{1}$

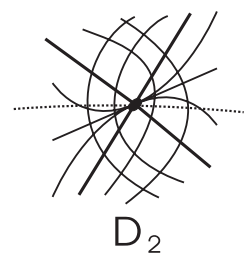

Fig. 2 - Principal curvature lines near a Darbouxian-like point on an umbilic curve, dotted. 
Proof. After division by $v$, the first order terms of differential equation of curvature lines, see equation (6), is given by:

$$
\begin{aligned}
\mathcal{F}(u, v,[d u: d v])= & -\left(A u+v+O_{1}(2)\right) d v^{2}+\left(2 u+B v+O_{2}(2)\right) d u d v \\
& +\left(A u+v+O_{3}(2)\right) d u^{2}
\end{aligned}
$$

Consider the Lie-Cartan line field $X=\left(\mathcal{F}_{p}, p \mathcal{F}_{p},-\left(\mathcal{F}_{u}+p \mathcal{F}_{v}\right)\right)$, where $p=d v / d u$. The singular points of $X$ along the projective line - represented by the $p$ axis - are defined by the cubic equation $R(p)=p^{3}+(A-B) p^{2}-3 p-A=0$. The condition $\Delta \neq 0$ means that $R$ has no multiple roots and consequently the singularities of $X$ restricted to the projective line are hyperbolic. The linear part of $X$ along the axis $p$ is given by:

$$
D X(0,0, p)=\left(\begin{array}{ccc}
2-2 A p & B-2 p & 0 \\
(2-2 A p) p & (B-2 p) p & 0 \\
0 & 0 & 3 p^{2}+2(A-B) p-3
\end{array}\right)
$$

The non vanishing eigenvalues of $D X(0,0, p)$ are

$$
\lambda_{1}=2+(B-2 A) p-2 p^{2}, \quad \lambda_{2}=3 p^{2}+2(A-B) p-3 .
$$

The resultant between $R$ and $\lambda_{2}=R^{\prime}$ is exactly $\Delta \neq 0$, while the resultant between $R$ and $\lambda_{1}$ is $(2-A B)\left(16+(2 a-b)^{2}\right)=\delta\left(16+(2 a-b)^{2}\right) \neq 0$.

Therefore in the hypothesis considered all the singular points of $X$ are hyperbolic. In the chart $(u, v, q=d u / d v)$ the Lie-Cartan line field $Z=\left(q \mathcal{F}_{q}, \mathcal{F}_{q},-\left(q \mathcal{F}_{u}+\mathcal{F}_{v}\right)\right)$ is regular at 0 .

Now consider the vector field $Y=\left(A u+v+O_{1}(2),-u-\frac{B}{2} v-O_{2}(2)\right)$. By properties of the index of umbilic points, see (Gutierrez and Sotomayor 1998), (Hopf 1979) and (Spivak 1980), it follows that $\operatorname{Ind}(Y, 0)=-2 \operatorname{Ind}\left(\mathcal{F}_{i}, 0\right)$, where $\mathcal{F}_{i},(i=1,2)$ denotes the principal foliations. As $\operatorname{det}(D Y(0))=(2-A B) / 2=\delta / 2$, it follows that $\operatorname{Ind}(Y, 0)=1$ provided $\delta>0$, and that $\operatorname{Ind}(Y, 0)=-1$ when $\delta<0$. Therefore, $\operatorname{Ind}\left(\mathcal{F}_{i}, 0\right)= \pm 1 / 2$.

In the hypothesis of item i), $\Delta<0$ and $\delta<0$, the field $X$ has only one hyperbolic saddle point and the index of the principal foliations is $1 / 2$ and so they define topologically a Darbouxian umbilic $D_{1}$, with one separatrix approaching the umbilic point and one hyperbolic sector for each principal foliation.

In the hypothesis of item ii), $\Delta>0$ and $\delta<0$, the field $X$ has two hyperbolic saddle points and one hyperbolic node. In this case the index of the principal foliations is $1 / 2$ and so is topologically a Darbouxian umbilic $D_{2}$, with two separatrices approaching the umbilic point, one hyperbolic sector and one parabolic sector for each principal foliation.

In the hypothesis of item iii), $\Delta>0$ and $\delta>0$, the field $X$ has three hyperbolic saddle points and the index of the principal foliations is $-1 / 2$ and they define topologically a Darbouxian umbilic $D_{3}$, with three separatrices approaching to the umbilic point and three hyperbolic sectors for each principal foliation. 
Figure 3 shows the behavior of $X$ near the projective line and the blowing down of the integral curves.

Clearly when $A \neq 0$ the umbilic separatrices are transversal to the curve of umbilic points which is parametrized by $v=0$.
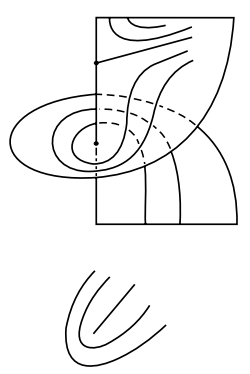
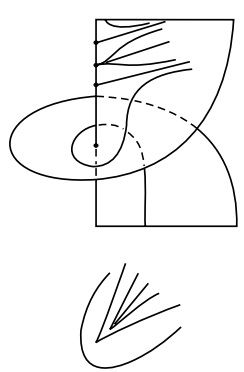
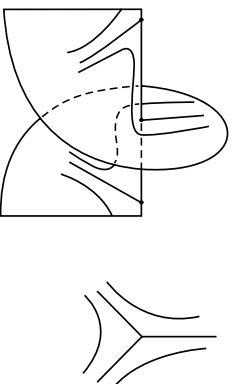

Fig. 3 - Behavior of $X$ in a neighborhood of the projective line and projection of the integral curves.

\section{SPHERICAL AND PLANAR UMBILIC CURVES}

In the previous sections have been studied a sample of the most generic situations, under the restriction on the surface of having an umbilic curve. Below will be considered the case where $k$ is a constant which implies the additional constrain that the umbilic curve be spherical or planar, a case also partially considered in (Carathéodory 1935). Under this double imposition the simplest patterns of principal curvature lines are analyzed in what follows.

Proposition 4. Let c be a regular closed spherical or planar curve. Suppose that $c$ is a regular curve of umbilic points on a smooth surface. Then the principal foliations near the curve are as follows.

i) If $\mathcal{H}_{v}(u, 0)=a(u) / 2 \neq 0$ and $a(u)>0$ for definiteness, then one principal foliation is transversal to the curve $c$ of umbilic points.

The other foliation defines a first return map (holonomy) $\pi$ along the oriented umbilic curve $c$, with first derivative $\pi^{\prime}=1$ and second derivative given by a positive multiple of

$$
\int_{0}^{l} k_{g}(u) \frac{a^{\prime}(u)}{a(u)^{\frac{3}{2}}} d u .
$$

When the above integral is non zero the principal lines spiral towards or away from c, depending on their side relative to $c$.

ii) If a (u) has only transversal zeros, near them the principal foliations have the topological behavior of a Darbouxian umbilic point $D_{3}$ at which a separatrix has been replaced with the umbilic curve. See Fig. 4. 

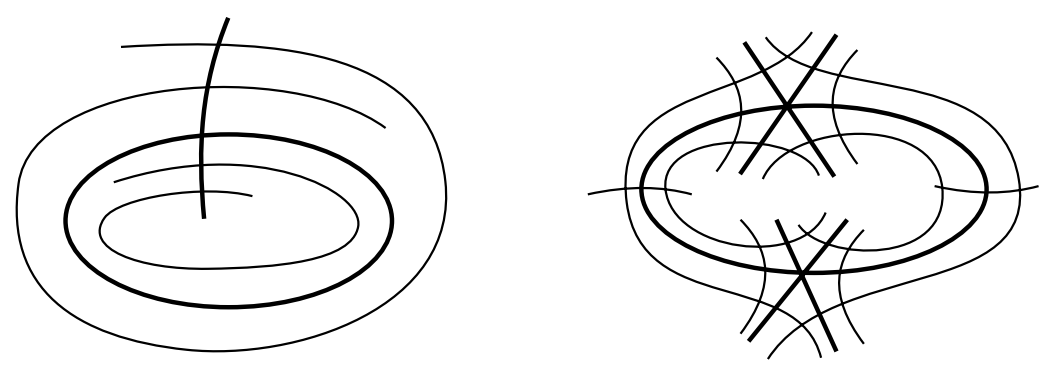

Fig. 4 -Curvature lines near a spherical umbilic curve.

PROOF. When $c$ is a spherical or planar curve it follows that $k(u)=k$. In the planar case $k=0$ and $k_{g}$ is the curvature of plane curves. In this case the differential equation of principal curvature lines, see equation (6), after division by $v$ is given by:

$$
\begin{aligned}
& -\left[\frac{1}{2} a^{\prime}(u) v+\frac{1}{6}\left(k_{g} a^{\prime}+b^{\prime}\right) v^{2}+O\left(v^{3}\right)\right] d v^{2} \\
& +\left[a(u)+\frac{1}{2}\left(b(u)-3 k^{3}-3 k_{g} a(u)\right) v\right. \\
& \left.+\frac{1}{6}\left(15 k^{3} k_{g}+\left(3 k_{g}^{2}-16 k^{2}\right) a(u)-a^{\prime \prime}-5 k_{g} b(u)\right) v^{2}+O\left(v^{4}\right)\right] d u d v \\
& +\left[\frac{1}{2} a^{\prime}(u) v+\frac{1}{6}\left(b^{\prime}-5 k_{g} a^{\prime}\right) v^{2}+O\left(v^{3}\right)\right] d u^{2}=0
\end{aligned}
$$

The curve $v=0$ is a solution of equation (7). As $a(u) \neq 0$ by hypothesis it follows that $v=0$ is a periodic orbit of (7). The Poincaré map is defined by $\pi\left(v_{0}\right)=v\left(l, v_{0}\right)$ where $v\left(u, v_{0}\right)$ is the solution of (7) with initial condition $v\left(0, v_{0}\right)=v_{0}$.

By the standard method of differentiation of solutions of differential equations with respect to initial conditions to obtain the Taylor expansion of $\pi$, it follows that $d v / d v_{0}(u)=\sqrt{\frac{a_{0}}{a(u)}}$, where $a_{0}=a(0)>0$ for definiteness. Therefore $\pi^{\prime}(0)=1$.

Differentiation shows that $q(u)=\frac{d^{2} v}{d v_{0}^{2}}(u, 0)$ satisfies the following linear differential equation:

$$
-\frac{1}{2} \frac{a_{0} a^{\prime} b(u)}{a(u)^{2}}+\frac{3}{2} \frac{a_{0} a^{\prime} k^{3}}{a(u)^{2}}-\frac{1}{6} \frac{a_{0} a^{\prime} k_{g}}{a(u)}+\frac{1}{3} \frac{a_{0} b^{\prime}}{a(u)}+a(u) q^{\prime}+\frac{1}{2} a^{\prime} q(u)=0 .
$$

Integration of the equation above leads to

$$
q(u)=\frac{a_{0}}{6} \frac{1}{\sqrt{a(u)}} \int_{0}^{u}\left[\frac{a^{\prime}}{a^{5 / 2}}\left(3 b(u)-9 k^{3}\right)+\frac{1}{a^{3 / 2}}\left(a^{\prime} k_{g}-2 b^{\prime}\right)\right] d u
$$

To obtain the second derivative of $\pi$, integration by parts gives:

$$
\pi^{\prime \prime}(0)=q(l)=\frac{1}{6} \sqrt{a_{0}} \int_{0}^{l} k_{g} \frac{a^{\prime}}{a^{3 / 2}} d u .
$$

This ends the proof of item i). 
To proceed consider the case where $a(u)$ has a transversal zero say at $u=0$. Therefore the differential equation of curvature lines, equation $(7)$, near $(0,0)$ can be written as:

$$
\mathcal{F}\left(u, v,[d u: d v]=[-v+\text { h.o.t }] d v^{2}+\left[2 u+a_{1} v+\text { h.o.t }\right]+[v+\text { h.o.t }] d u^{2}=0,\right.
$$

where $a_{1}=\left(b(0)-3 k^{3}\right) / a^{\prime}(0)$. Consider the Lie-Cartan line field $X=\left(\mathcal{F}_{p}, p \mathcal{F}_{p},-\left(\mathcal{F}_{u}+p \mathcal{F}_{v}\right)\right)$, where $p=d v / d u$. The field $X$ has three real singular points along the projective line - axis $p$ and its are defined by the polynomial equation $p\left(p^{2}-a_{1} p-3\right)=0$. The linear part of $X$ along the axis $p$ is given by:

$$
D X(0,0, p)=\left(\begin{array}{ccc}
2 & a_{1}-2 p & 0 \\
2 p & \left(a_{1}-2 p\right) p & 0 \\
0 & 0 & 3 p^{2}-2 a_{1} p-3
\end{array}\right)
$$

The eigenvalues of $D X(0,0, p)$ are $\lambda_{1}=2-2 p^{2}+a_{1} p$ and $\lambda_{2}=3 p^{2}-2 a_{1} p-3$.

At $p=0$ it follows that $\lambda_{1} \lambda_{2}=-6<0$ and so $(0,0,0)$ is a hyperbolic saddle point of $X$. Evaluating $\lambda_{1}$ and $\lambda_{2}$ at the other singular points $\left(0,0, p_{i}\right)$ of $X$ it is obtained that $\lambda_{1}=-\left(1+p_{i}^{2}\right)$ and $\lambda_{2}=3+p_{i}^{2}$. Therefore these two singular points of $X$ are also hyperbolic saddle points. Near the projective line the phase portrait of $X$ is as illustrated in Fig. 5. Blowing down the phase portrait of $X$ it follows that the singular point is equivalent to a Darbouxian umbilic point of type $D_{3}$.
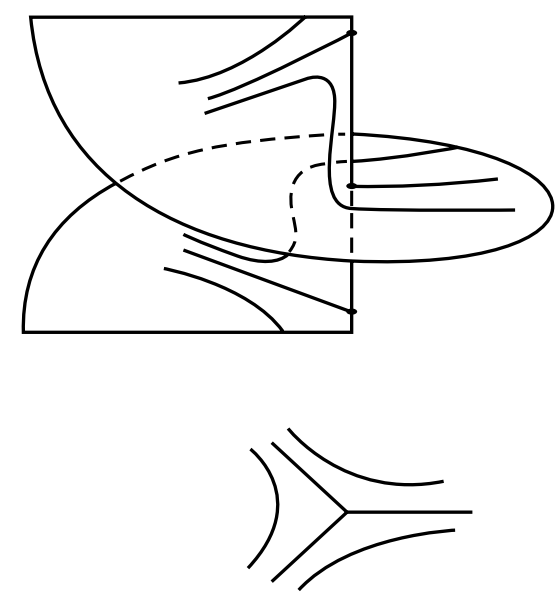

Fig. 5 - Resolution of $X$ near the projective line.

REMARK 1 . By properly choosing functions $k_{g}$ and $a(u)=2 \mathcal{H}_{v}$ it is possible to construct explicit examples of the spiraling behavior in item i) of Proposition 4.

Proposition 5. A closed regular curve $c:[0, l] \rightarrow \mathbb{R}^{3}$ parametrized by arc length $u$ is a curve of umbilic points of a regular surface containing $c$ if and only if $\int_{0}^{l} \tau(u) d u \in 2 k \pi, k \in \mathbb{Z}$. 
Proof. The Frenet frame of $c$ is given by $\{t, n, b\}$ and the following equations holds:

$$
\begin{aligned}
t^{\prime} & =k n \\
n^{\prime} & =-k t+\tau b \\
b^{\prime} & =-\tau n
\end{aligned}
$$

For any regular surface containing $c$ the Darboux frame $\{t, N \wedge t, N\}$ and the Frenet frame $\{t, n, b\}$ are related by:

$$
\begin{gathered}
N=\cos \theta(u) n+\sin \theta(u) b \\
N \wedge t=-\sin \theta(u) n+\cos \theta(u) b .
\end{gathered}
$$

Direct calculation shows that $k_{n}(u)=k(u) \cos \theta(u), \quad k_{g}(u)=-k(u) \sin \theta(u)$ and $\tau_{g}(u)=$ $-\left(\theta^{\prime}(u)+\tau(u)\right)$.

Supposing that $c$ is a curve of umbilic points it follows, see Proposition 1, that $\tau_{g}=0$ and $k_{n}(c(u), T)=k_{n}(c(u), N \wedge T)=k(u) \cos \theta(u)$.

Therefore in order to obtain a regular surface containing $c$ (closed curve) it is necessary that $\int_{0}^{l} \tau(u) d u$ be an integer multiple of $2 \pi$. Clearly, from equation (2) this condition is also sufficient.

The following corollary is the first case discussed in (Carathéodory 1935).

COROLLARY 1. Any regular spherical closed curve c is a curve of umbilic points of a regular surface which contains $c$.

Proof. For any closed spherical curve it follows that $\tau_{g}=0$ and therefore $\int_{0}^{l} \tau(u) d u=$ $-\int_{0}^{l} \theta^{\prime}(u) d u=0$.

\section{CONCLUDING REMARKS}

The interest on the structure of principal lines in a neighborhood of a continuum of umbilic points, forming a curve, in an analytic surface goes back to the work of Carathéodory (Carathéodory 1935). For previous results related to this subject, Carathéodory refers in his paper to the books of Monge (Monge 1850) and Dupin (Dupin 1813), reliquae not found by the authors.

In the present work has been carried out an independent, explicit, self sufficient and constructive study of umbilic curves on smooth surfaces, that holds also for regular closed curves. Here only the simplest, least degenerate cases, have been considered. A partial comparison of the results of this paper with those of (Carathéodory 1935) is attempted below.

In (Carathéodory 1935) Carathéodory established that any local analytic regular arc of curve in $\mathbb{R}^{3}$ is a curve of umbilic points of a piece of analytic surface. Proposition 5, which also holds in the analytic case, gives a global independent version. 
In some cases Carathéodory also determined the behavior of principal lines near the curve of umbilic points. The transversal case in Proposition 2 can be found in his paper. The tangential case is not there.

The cases studied in Proposition 3 are not treated in (Carathéodory 1935).

Concerning Proposition 4, case $i$ ), which gives an explicit criterion for quadratic spiraling approach to the umbilic closed curve, it seems that Carathéodory was aware of the possibility of spiraling, but gave no criterion or example for this situation. Case $i i$ ) in Proposition 4 was not treated in his paper.

In (Carathéodory 1935) the focal surfaces - caustics - received great attention. In fact Carathéodory starts his analysis with the focal surfaces from which he obtains the surface with an umbilic curve. In the present direct approach, the focal surfaces can be obtained from their standard expression

$$
\alpha+r_{i} N
$$

where $r_{i}=\left(k_{i}\right)^{-1}, i=1,2$ are the curvature radii defined in terms of the principal curvatures $k_{2,1}=\mathcal{H} \pm \sqrt{\mathcal{H}^{2}-\mathcal{K}}$, expressed in function of the Mean and Gaussian curvatures given in equation (4).

Additional analysis - complemented with some plotting - must be done to fully grasp the diversity of focal surfaces possible for surfaces with an umbilic curve exhibiting the several points studied here. In (Carathéodory 1935) only the first cases in Propositions 2 and 4 seem to have been considered.

\section{ACKNOWLEDGMENTS}

The authors are fellows of Conselho Nacional de Desenvolvimento Científico e Tecnológico (CNPq). This work was done under the project $\mathrm{CNPq} /$ Programa de Apoio ao Desenvolvimento Científico e Tecnológico/PADCT 620029/2004-8. The second author acknowledges the help of P. Tonelli and E. Harle in translating (Carathéodory 1935).

\section{RESUMO}

Neste trabalho estuda-se o comportamento das linhas de curvatura principal próximas a uma curva de pontos umbílicos, numa superfície suave.

Palavras-chave: ponto umbílico, linhas de curvatura principal, ciclos principais.

\section{REFERENCES}

Carathéodory C. 1935. Einfach Bemrkungen uber Nabelpunktscurven. Lecture at Breslau, Complete Works 5: 26-30.

Darboux G. 1896. Leçons sur la Théorie des Surfaces, vol. IV Sur la forme des lignes de courbure dans la voisinage d'un ombilic, Note 07, Gauthier Villars, Paris. 
Dupin CH. 1813. Développements de Géometrie, Paris, Courcier.

Garcia R AND Sotomayor J. 1997. Structural stability of parabolic points and periodic asymptotic lines, Matemática Contemporânea 12: 83-102.

Garcia R And Sotomayor J. 2000. Lines of Axial Curvature on Surfaces Immersed in $\mathbb{R}^{4}$, Differential Geometry and its Applications 12: 253-269.

Garcia R, Gutierrez C and Sotomayor J. 2000. Lines of Principal Curvature around Umbilics and Whitney Umbrellas, Tohoku Math J 52: 163-172.

Garcia R, Gutierrez C and Sotomayor J. 2004. Bifurcations of Umbilic Points and related Principal Cycles. J Dyn Differ Equ 15 (in press).

Gutierrez C and Sotomayor J. 1982. Structural Stable Configurations of Lines of Principal Curvature, Asterisque 98-99: 185-215.

Gutierrez C And Sotomayor J. 1991. Lines of Curvature and Umbilic Points on Surfaces, Brazilian $18^{\text {th }}$ Math. Coll., IMPA, Reprinted as Structurally Configurations of Lines of Curvature and Umbilic Points on Surfaces, Monografias del IMCA.

Gutierrez C and Sotomayor J. 1998. Lines of Curvature, Umbilical Points and Carathéodory Conjecture, Resenhas IME-USP 03: 291-322.

Hopf E. 1979. Differential Geometry in the Large, Lect. Notes in Math. 1000, Springer Verlag.

Monge G. 1850. Application de l'Analyse à la Géométrie, Bachalier, Paris, Reimpression by University Microfilms International, Ann Arbor, MI.

SpIVAK M. 1980. A Comprehensive Introduction to Differential Geometry, Vol. III, Publish or Perish, Berkeley. 